\title{
Supersolid states in a hard-core Bose-Hubbard model on a layered triangular lattice
}

\author{
Ryota Suzuki and Akihisa Koga \\ Department of Physics, Tokyo Institute of Technology, Tokyo 152-8551, Japan
}

(Dated: November 4, 2018)

\begin{abstract}
We study ground-state properties in a hard-core Bose-Hubbard model on a layered triangular lattice. Combining cluster mean-field theory with the density matrix renormalization group method, we discuss the effect of the interlayer coupling on the supersolid states realized in a single layered model. By examining the distributions for the particle density and superfluid order parameter, the rich phase diagram of the system is obtained. We find that the supersolid states are widely stabilized at a commensurate filling, in contrast to the case of the single layered model. The nature of the supersolid states is also addressed.
\end{abstract}

\section{INTRODUCTION}

Ultracold bosonic systems have attracted much interest since the successful observation of Bose-Einstein condensation in ${ }^{87} \mathrm{Rb}$ atoms [1]. One of the interesting topics in this field is an optical lattice system [2 [5], which is formed by loading the ultracold atoms in a periodic potential. This gives us clean correlated bosonic systems on various lattices with controllable parameters. In fact, remarkable phenomena have been observed such as the phase transition between Mott insulating and superfluid states on cubic [6] and triangular lattices [7]. Recently, the possibility of a supersolid state, where solid and superfluid states coexist, has been discussed. The existence of the supersolid state has experimentally been suggested in the ${ }^{4} \mathrm{He}$ system [8], and has theoretically been discussed in strongly correlated systems such as bosonic 9 20] and fermionic 21 24] systems and Bose-Fermi mixtures [25, 26]. As for a hard-core bosonic model, it has been clarified that lattice geometry as well as intersite correlations play a key role in stabilizing the supersolid state 12 14.

The hard-core Bose-Hubbard model on a triangular lattice has been discussed in detail [12, 13, 16, 17, 19]. In the weak coupling region, the superfluid state is realized. On the other hand, in the strong coupling region, the solid state appears at a one-third (two-thirds) filling, where one of three sites is filled (empty) in a $\sqrt{3} \times \sqrt{3}$ ordering with wave vector $\mathbf{Q}_{2}=(4 \pi / 3,0)$ [27]. Between the superfluid and solid states, the supersolid state is realized around half-filling, where a part of particles crystallize and the others form the superfluid state [13]. By contrast, no such supersolid states have been studied in the Bose-Hubbard model on layered triangular lattice. This should be crucial to observe the supersolid state in the realistic optical lattice systems [28]. An important point is that the supersolid state on each layer has threefold degeneracy for particle distributions. Therefore, it is desired to discuss how the interlayer couplings affect the stability of the supersolid state.

To clarify this point, we systematically study ultracold bosons in an optical lattice with the layered triangular structure, which is schematically shown in Fig. 10(a). We discuss ground-state properties of this model, combining (a)

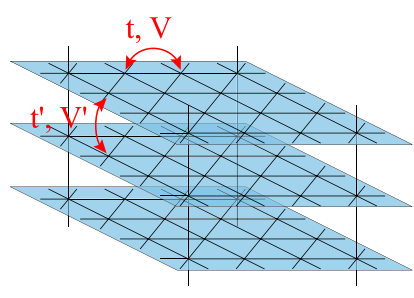

(b)

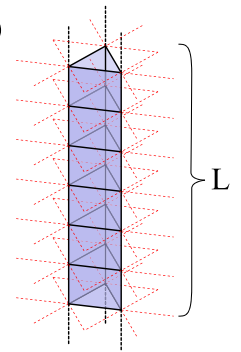

FIG. 1: (Color online) (a) Bose-Hubbard model on layered triangular lattices. (b) The effective cluster model with a tube structure, which are treated in the framework of cluster mean-field theory.

cluster mean-field (CMF) theory [29] with the density matrix renormalization group (DMRG) method 30, 31]. The rich phase diagram is obtained where the solid and supersolid states with various particle distributions compete with the superfluid state. We demonstrate that the supersolid state is realized even at half filling, in addition to the well-known supersolid states discussed in a single layered model [13]. We also discuss the nature of the supersolid states.

The paper is organized as follows. In $₫$ II we introduce the Bose-Hubbard model for the layered optical lattice system and explain the possible particle distributions for the solid and supersolid states. We briefly outline our theoretical approach. In \III we study ground-state properties in the layered system and discuss the stability of the supersolid states. A summary is given in the final section.

\section{MODEL AND METHOD}

We consider ground-state properties of interacting bosons on the layered triangular lattice. Here, we assume sufficiently large onsite interactions. In the case, the system should be described by the following hardcore Bose-Hubbard model as,

$$
H=-\sum_{\langle i, j\rangle} t_{i j}\left(a_{i}^{\dagger} a_{j}+\text { h.c. }\right)+\sum_{\langle i, j\rangle} V_{i j} n_{i} n_{j}
$$


where $\langle i, j\rangle$ denotes the summation over nearest neighbor sites and $a_{i}^{\dagger}\left(a_{i}\right)$ is the creation (annihilation) operator of a boson at site $i$. The hopping $t_{i j}=t\left(t^{\prime}\right)$ and the intersite interaction $V_{i j}=V\left(V^{\prime}\right)$ when the sites $i$ and $j$ are located in the same (distinct) layer. This model is symmetric when interchanging particles with holes. The symmetric condition is given by $\mu / V=3+r$, where $\mu$ is the chemical potential and $r=V^{\prime} / V$. In the paper, we restrict our discussions to the system with $\mu / V \leq 3+r$, where the particle density $n\left(=\sum_{i}\left\langle n_{i}\right\rangle / N\right) \leq 1 / 2$ and $N$ is the total number of sites.

When the interlayer couplings are zero $\left(t^{\prime}=V^{\prime}=0\right)$, the model Hamiltonian eq. (10) is reduced to the hardcore Bose-Hubbard model on a single-layer triangular lattice [13]. In the limit, it is known that the supersolid states are realized, which are surrounded by the superfluid state in the weak coupling region and solid states with $n=1 / 3$ and $2 / 3$ in the strong coupling region [13]. Note that at the particle-hole symmetric case, two supersolid states with distinct particle density are degenerate. Therefore, when the system is fixed at half filling, the genuine supersolid state is not stable, but the phase separation between two supersolid states appears in the strong coupling region. When $t=V=0$, the model is reduced to the one-dimensional Bose-Hubbard model, where no supersolid state appears [32, 33]. In the paper, we study the hard-core Bose-Hubbard model on the layered triangular lattice to discuss how the supersolid states is realized.

First, we define the order parameters for the possible ground states. In the layered system with intersite interactions, various type of particle distributions are naively expected. In fact, in the simple (classical) limit with

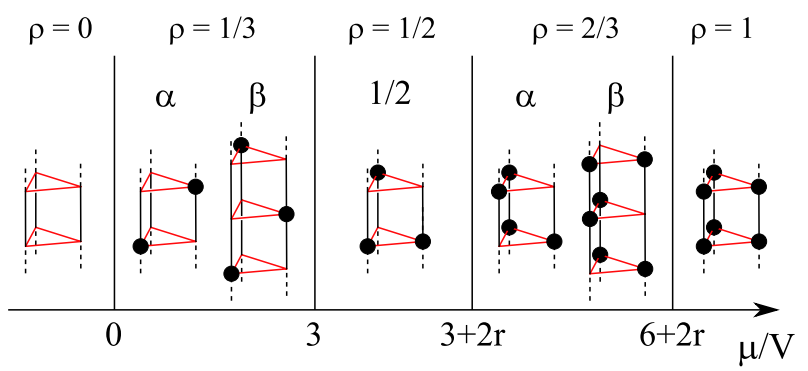

FIG. 2: The density profiles of solid states realized in strong coupling limit.

$t=t^{\prime}=0$, a phase diagram should be obtained, as shown in Fig. 2. When $\mu / V<0(6+2 r<\mu / V)$, an empty (fully-occupied) state appears. When $0<\mu / V<3$ $(3+2 r<\mu / V<6+2 r)$, the solid state appears with $n=1 / 3(n=2 / 3)$. If one focuses on a certain layer, the solid state has three-fold degeneracy. This means that the layered system has a large residual entropy in this limit. Here, we specify the solid structure with a unit cell composed of six and nine sites as $\alpha$ - and $\beta$-type solids, respectively (see Fig. 2). Note that we did not find any particle distributions with larger periods when the hoppings $t, t^{\prime}$ are introduced. The $\alpha$ - and $\beta$-type particle distributions are characterized by the following quantities as

$$
\begin{aligned}
& \rho_{2}=\left|\rho\left(\frac{4 \pi}{3}, 0, \pi\right)\right|, \\
& \rho_{3}=\left|\rho\left(\frac{4 \pi}{3}, 0,+\frac{4 \pi}{3}\right)\right|+\left|\rho\left(\frac{4 \pi}{3}, 0,-\frac{4 \pi}{3}\right)\right|,
\end{aligned}
$$

where

$$
\rho(\mathbf{q})=\frac{1}{N} \sum_{i}\left\langle n_{i}\right\rangle e^{-\mathbf{q} \cdot \mathbf{r}_{i}}
$$

On the other hand, at half filling $(n=1 / 2)$, a different particle distribution appears. Fig. 2 shows that the particle density in each layer alternate in the $z$-direction. The state is characterized by the following quantity, in addition to $\rho_{2}$, as

$$
\Delta n=|\rho(0,0, \pi)|
$$

Here, we specify this particle distribution as the " $1 / 2$ "type one, for simplicity. In the weak coupling limit, the superfluid (SF) state is naively expected. The order parameter is defined as

$$
\Psi=\frac{1}{N} \sum_{i}\left\langle a_{i}\right\rangle
$$

When the system has both $\Psi$ and some of the other parameters defined above, there appears the coexistence between the superfluid and particle density wave, and we can say that the supersolid state is realized. For simplicity, we specify the solid (supersolid) states as the S- $\alpha$, S- $\beta$, and S- $1 / 2$ (SS- $\alpha$, SS- $\beta$, and SS- $1 / 2$ ), corresponding to the particle distributions. The relation between the ordered states and quantities are summarized in Table 【.

\begin{tabular}{c|ccc|ccc|c} 
& S- $\alpha$ & S- $\beta$ & S- $-1 / 2$ & SS- $\alpha$ & SS- $\beta$ & SS- $1 / 2$ & SF \\
\hline$\rho_{2}$ & $\neq 0$ & 0 & $\neq 0$ & $\neq 0$ & 0 & $\neq 0$ & 0 \\
$\rho_{3}$ & 0 & $\neq 0$ & 0 & 0 & $\neq 0$ & 0 & 0 \\
$\Delta n$ & 0 & 0 & $\neq 0$ & 0 & 0 & $\neq 0$ & 0 \\
$\Psi$ & 0 & 0 & 0 & $\neq 0$ & $\neq 0$ & $\neq 0$ & $\neq 0$
\end{tabular}

TABLE I: The order parameters of the possible ground states

To discuss ground-state properties in the hardcore Bose-Hubbard model, we make use of the CMF method [29]. In the method, the original lattice model is mapped to an effective cluster model, where particle correlations in the cluster can be taken into account properly. The expectation values of the intercluster Hamiltonian are obtained via a self-consistency condition imposed on the effective cluster problem. This method has an advantage in discussing quantum phase transitions correctly since not only stable states but also metastable states can be treated. Therefore, the CMF method 
has successfully been applied to the quantum spin systems [29, 34] and bosonic systems [15, 19, 20].

In the study, we consider the three-leg tube model with the length $L$ as the effective cluster model, as shown in Fig. 1 (b). The effective Hamiltonian is explicitly given as,

$$
\begin{aligned}
H_{\mathrm{CMF}} & =\sum_{C} H_{\mathrm{CMF}}^{C}, \\
H_{\mathrm{CMF}}^{C} & =-\sum_{\langle i, j\rangle_{C}} t_{i j}\left(a_{i}^{\dagger} a_{j}+\text { h.c. }\right)+\sum_{\langle i, j\rangle_{C}} V_{i j} n_{i} n_{j} \\
& -\sum_{\langle i, j\rangle_{C}^{\prime}} t_{i j}\left(\left\langle a_{j}\right\rangle a_{i}^{\dagger}+\text { h.c. }\right)+\sum_{\langle i, j\rangle_{C}^{\prime}} V_{i j}\left\langle n_{j}\right\rangle n_{i} \\
& -\mu \sum_{i \in C} n_{i}+E_{0} \\
E_{0} & =\frac{1}{2} \sum_{\langle i, j\rangle_{C}^{\prime}}\left[t_{i j}\left(\left\langle a_{i}\right\rangle^{*}\left\langle a_{j}\right\rangle+\text { c.c. }\right)-V_{i j}\left\langle n_{i}\right\rangle\left\langle n_{j}\right\rangle\right]
\end{aligned}
$$

where the symbol $\langle i, j\rangle_{C}\left[\langle i, k\rangle_{C}^{\prime}\right]$ denote the summation over nearest neighbor sites in the cluster (between clusters). Note that this three-leg tube is too thin to quantitatively discuss quantum phase transitions in the system [19]. However, it is known that an effective three-site cluster in a single layer reproduce the reasonable phase diagram. An important point in this study is that the cluster with a large value of $L$ is necessary to discuss the stability of the solid and supersolid states with longperiod structures. To this end, we here make use of the DMRG technique as an effective cluster solver [20]. It is known that this method is powerful for the onedimensional systems [30, 31]. Furthermore, by combining the DMRG method with a mean-field theory, the phase transitions in higher dimensions have been discussed for the Heisenberg models [35, 36] and fermionic Hubbard models 37, 38]. Some details of the CMF+DMRG method are explained in Ref. [20]. In the following, we take the intralayer interaction $(V)$ as unit of energy and fix the ratio of interactions as $r=1 / 2$.

\section{RESULTS}

In the section, we discuss ground state properties in the hard-core Bose-Hubbard model on the layered triangular lattice. We then clarify the role of the interlayer coupling in stabilizing the supersolid state in the system.

\section{A. Low density case}

First, we consider the hard-core bosonic system with a low particle density, fixing the chemical potential as $\mu / V=0.68(3+r)$. Combining the CMF method with the DMRG, we obtain the order parameters for the system with a fixed ratio $t / V=0.1$, as shown in Fig. 3. In

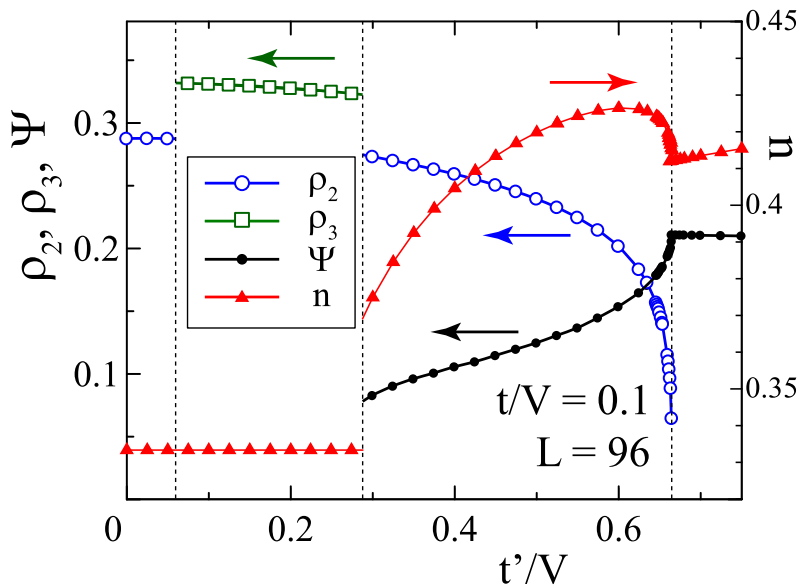

FIG. 3: (Color online) The order parameters $\rho_{2}$ (open circles), $\rho_{3}$ (open squares), the superfluid order parameter $\Psi$ (solid circles), and the particle density $n$ (solid triangles) as a function of $t^{\prime} / V$ in the system with $t / V=0.1$ and $V^{\prime} / V=0.5$.

this case, the particle density in the layer is always uniform in the $z$-direction $(\Delta n=0)$. When $t^{\prime} / V=0$, the particle density is one-third $(n=1 / 3)$, the order parameter $\rho_{2}$ is finite and the other order parameters are zero. This means that the S- $\alpha$ state is realized in the limit. The introduction of $t^{\prime}$ little decreases $\rho_{2}$ and the S- $\alpha$ state still remains. At $t^{\prime} / V=\left(t^{\prime} / V\right)_{c 1}$, the particle density is not changed, but $\rho_{2}$ suddenly vanishes and $\rho_{3}$ appears instead, where $\left(t^{\prime} / V\right)_{c 1} \sim 0.06$. This implies that the particle distributions are drastically changed, and the $\mathrm{S}-\beta$ state is realized. Further increase in the hopping induces another first-order phase transition at $t^{\prime} / V=\left(t^{\prime} / V\right)_{c 2}$, where $\left(t^{\prime} / V\right)_{c 2} \sim 0.29$. In the case, both $\rho_{2}$ and $\Psi$ are induced instead of $\rho_{3}$ and the particle number becomes away from the commensurate filling $(n \neq 1 / 3)$. Therefore, we can say that the coexisting state with the $\alpha$-type particle distribution (SS- $\alpha$ ) is realized. Increasing the hopping $t^{\prime}$, superfluid correlations are enhanced and the $\alpha$-type particle distribution becomes obscure, as shown in Fig. 3. At $t^{\prime} / V=\left(t^{\prime} / V\right)_{c 3}$, the first-order phase transition occurs with small jumps in the order parameters and the particle density, where $\left(t^{\prime} / V\right)_{c 3} \sim 0.66$. In the large $t^{\prime}$ region, no spatial distribution appears in the particle density and the genuine superfluid state is realized.

To discuss how the $\alpha$-type state competes with the $\beta$ type one, we also show the ground state energy in Fig. 4. The energy for each state is smoothly varied when the interlayer hopping is varied. We find that these curves intersect each other at two points $\left(t^{\prime} / V\right)_{c 1}$ and $\left(t^{\prime} / V\right)_{c 2}$, where the first-order quantum phase transitions occur. If we focus on the restricted space specified by the $\alpha$ type ( $\beta$-type) particle distribution, we find the critical point between the solid and supersolid states at $\left(t^{\prime} / V\right)_{c} \sim$ $0.23(0.40)$, which is shown as a solid circle in Fig. 4. However, the corresponding energy is higher than the ground-state one, which means that the quantum phase 


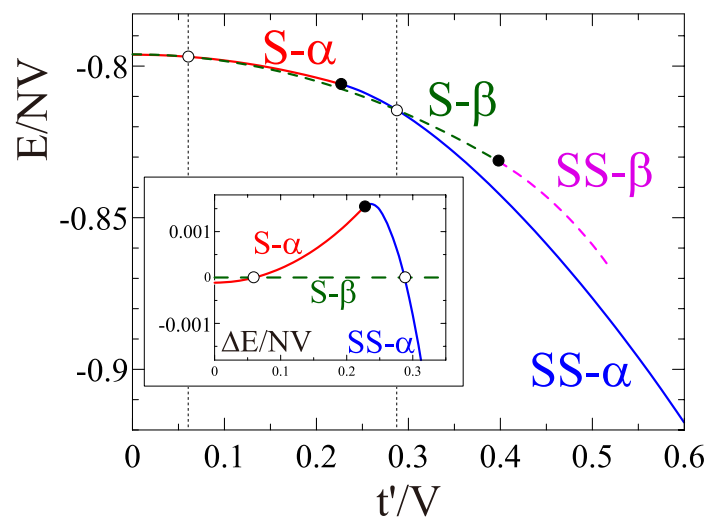

FIG. 4: (Color online) The energies for S- $\alpha$, S- $\beta$, SS- $\alpha$ and SS- $\beta$ states in the system with $t / V=0.1$ and $r=0.5$. Open circles represent the first-order transition points and solid ones represent the second-order transition points in metastable states. Inset shows the energy difference $\Delta E=E_{i}-E_{S-\beta}$, where $i=\mathrm{S}-\alpha$ and SS $-\alpha$.

transition occurs in the metastable state. Therefore, we could not find critical behavior in the ground state when $t / V=0.1$.

By performing similar calculations for different values of $t / V$, we obtain the zero-temperature phase diagram as shown in Fig. 5. In the weak coupling region, the

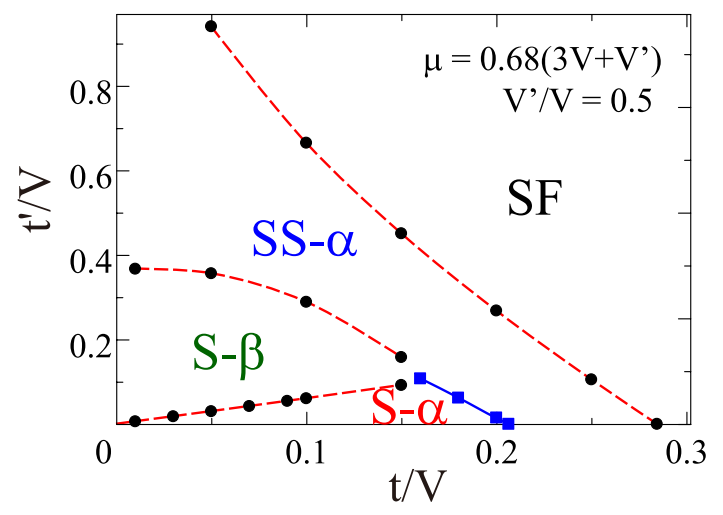

FIG. 5: (Color online) The zero temperature phase diagram for the system with $r=0.5$ and $\mu / V=0.68(3+r)$. The solid (dashed) lines represent the phase boundary, where the first-order (second-order) transition occurs.

superfluid state appears with a finite $\Psi$. Increasing the interactions $\left(V, V^{\prime}\right)$, translational symmetry is broken in addition to $U(1)$ symmetry and the $\alpha$-type supersolid state is realized. Further increase of the interactions yields two solid phases (S- $\alpha$ and S- $\beta$ ) with $n=1 / 3$. We find that the phase transition between the solid and supersolid states with the $\alpha$-type particle distribution is of second-order and the others are of first-order.

There are two remarkable points in the phase diagram. One of them is that the $\mathrm{S}-\beta$ state is stable against the $\mathrm{S}-\alpha$ and SS- $\alpha$ states. Since the S- $\alpha$ and S- $\beta$ states are degenerate in the classical limit $\left(t=t^{\prime}=0\right)$, the competition should be understood by the strong coupling expansion. The energies for the $\mathrm{S}-\alpha$ and $\mathrm{S}-\beta$ states are expressed by the second-order perturbation theory as

$$
\begin{aligned}
& \frac{E_{S-\alpha}}{N V} \sim-\frac{1}{3}\left[\frac{2}{3+r}\left(\frac{t^{\prime}}{V}\right)^{2}+\frac{3}{2} \frac{2+r}{1+r}\left(\frac{t}{V}\right)^{2}\right] \\
& \frac{E_{S-\beta}}{N V} \sim-\frac{1}{3}\left[\frac{2}{3}\left(\frac{t^{\prime}}{V}\right)^{2}+\frac{6}{2+r}\left(\frac{t}{V}\right)^{2}\right]
\end{aligned}
$$

This means that the $\alpha$-type solid state is mainly stabilized by the intralayer hopping while the $\beta$-type solid state is by the interlayer hopping. The first-order phase transition between two solid states occurs at a certain point

$$
\left(t^{\prime} / t\right)_{c}=\frac{3}{2} \sqrt{\frac{3 r+r^{2}}{2+3 r+r^{2}}} .
$$

This is consistent with the phase boundary in the phase diagram although a quantitative difference appears due to a thin tube structure of the effective cluster. For the above reason, the $\mathrm{S}-\beta$ state is realized in the strong coupling region with $t^{\prime} / t>\left(t^{\prime} / t\right)_{c}$.

The other point is that the $\mathrm{SS}-\alpha$ state is always stable against the $\mathrm{SS}-\beta$ state, in contrast to the competition between the solid states discussed above. This may be explained by considering the nature of the supersolid states. The supersolid state is the coexistence between the solid state with a certain particle distribution and the superfluid state. Therefore, the stability of the supersolid state with $n>1 / 3$ is roughly discussed in terms of the effective model, which is composed of localized particles and itinerant "defect" particles [16]. In this case, the defect particles can hop on layered honeycomb lattices, as shown in Fig. 6. If the particle gains the kinetic
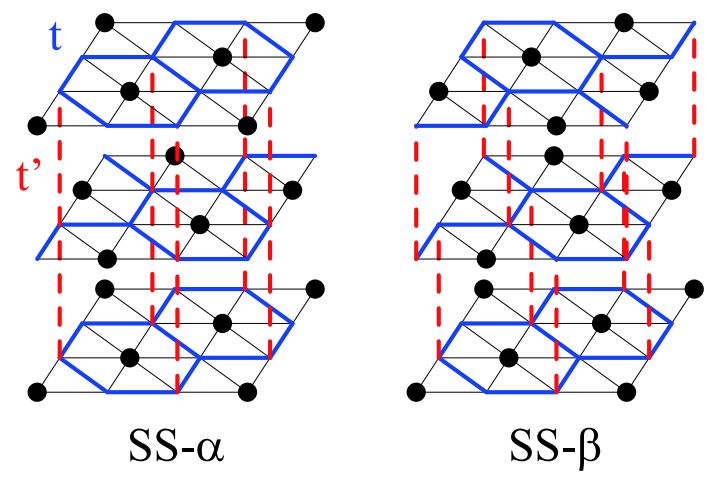

FIG. 6: Schematic description of the effective model for the supersolid states with $\alpha$ and $\beta$ structures. Solid circles represent localized particles. Bold solid and dashed lines represent the hoppings $t$ and $t^{\prime}$ for the defect particles.

energy against the intersite repulsion, particles are spontaneously doped. This induces the coexistence between the Bose-Einstein condensation for defect particles and the solid state for localized particles, that is, the supersolid state is realized. By using the first-order perturbation, we obtain the single particle energy for each state 
as

$$
\begin{aligned}
& \epsilon_{S S-\alpha}=-\tilde{\mu}-t^{\prime}-\sqrt{(3 t)^{2}+\left(t^{\prime}+V^{\prime}\right)^{2}} \\
& \epsilon_{S S-\beta}=-\tilde{\mu}-t^{\prime}-3 t
\end{aligned}
$$

where $\tilde{\mu}=\mu-3 V-V^{\prime}$. The relation $\epsilon_{S S-\alpha}<\epsilon_{S S-\beta}$ is always satisfied, implying that the $\mathrm{SS}-\alpha$ state is more favorable due to a one-dimensional network along the $z$ direction. This result is consistent with the fact that $\mathrm{SS}-\beta$ state never appears in the ground-state phase diagram (see Fig. 5). We also find that the phase boundary between the SS- $\alpha$ and S- $\alpha$ states roughly obtained from $\epsilon_{S S-\alpha}=0$ is consistent with that in the phase diagram (not shown).

In the section, we have clarified that the $\mathrm{SS}-\alpha$ state is indeed realized at the low particle density. As discussed above, the nature of the $\mathrm{SS}-\alpha$ state is essentially the same as that of the supersolid state in the single layered model. Therefore, we can say that the supersolid state realized in the single layer is stable against the interlayer coupling.

\section{B. Half filling}

Here, we consider the hard-core bosonic system at the symmetric condition with $\mu / V=3+r$ to clarify how the supersolid state is stabilized. Fig. 7 shows the order parameters for the system with a fixed ratio $t / V=0.1$. In this case, the system is always half-filled $(n=1 / 2)$

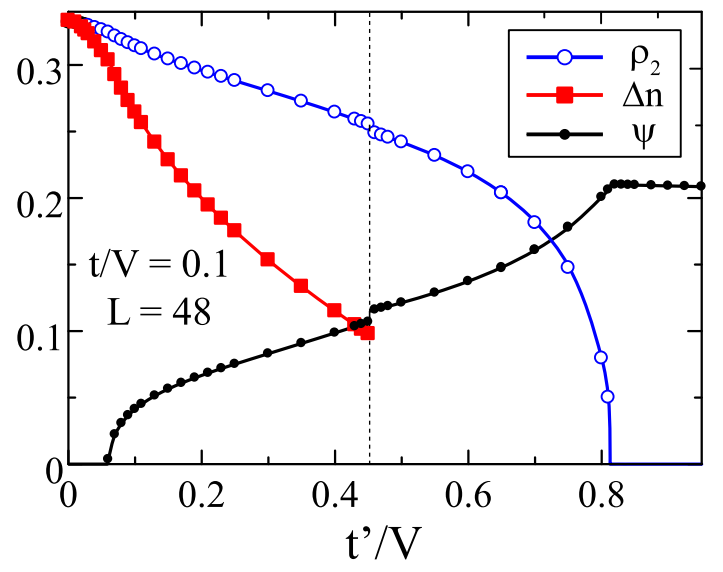

FIG. 7: (Color online) The order parameters $\rho_{2}$ (open circles), $\Delta n$ (solid squares), and the superfluid order parameter $\Psi$ (solid circles) as a function of $t^{\prime} / V$ in the system with $t / V=0.1, r=0.5$ and $\mu / V=3+r$.

and $\rho_{3}$ is zero. Therefore, the $\beta$-type particle distribution is not realized at half filling. When $t^{\prime} / V<\left(t^{\prime} / V\right)_{c 1}$, $\rho_{2}$ and $\Delta n$ are finite and $\Psi=0$, where $\left(t^{\prime} / V\right)_{c 1} \sim 0.06$. This implies that the $\mathrm{S}-1 / 2$ state is realized. Increasing the hopping $t^{\prime}$, the second-order phase transition occurs to the SS- $1 / 2$ state at $t^{\prime} / V=\left(t^{\prime} / V\right)_{c 1}$, where $\Psi$ is induced. A further increase in the hopping $t^{\prime}$ increases $\Psi$ and decreases $\rho_{2}$ and $\Delta n$. At the transition point, $\Delta n$ suddenly vanishes and the phase transition occurs to the
SS- $\alpha$ state, where the small jumps appear in the curves of $\rho_{2}$ and $\Psi$. The first-order transition point is determined as $\left(t^{\prime} / V\right)_{c 2}=0.45$, by deducing the crossing point of the energy curves for competing states (not shown). The increase in the hopping $t^{\prime}$ decreases $\rho_{2}$ and increases $\Psi$. Finally, $\rho_{2}$ vanishes and the second-order phase transition occurs to the superfluid state at a critical point $\left(t^{\prime} / V\right)_{c 3}$, where $\left(t^{\prime} / V\right)_{c 3} \sim 0.81$.

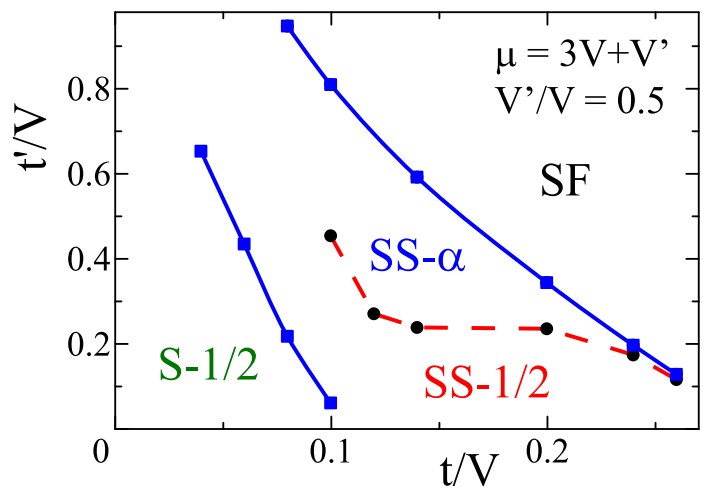

FIG. 8: (Color online) The zero temperature phase diagram for the half filled system with $r=0.5$. The solid (dashed) lines represent the phase boundary, where the second- (first-)order phase transition occurs.

We perform the CMF calculations for several values of $t / V$ and end up with the phase diagram at half filling, as shown in Fig. 8. In the strong coupling region, the $\mathrm{S}-1 / 2$ state is realized, where alternating behavior appears in the local particle density. The increase in the hoppings $t$ and $t^{\prime}$ enhances superfluid correlations and the quantum phase transition occurs. The SS- $1 / 2$, SS- $\alpha$, and superfluid states are realized, depending on the parameters. When $t / V$ is small and $t^{\prime} / V$ is large, the obtained order parameters strongly depend on the tube length $L$ in the $\mathrm{CMF}+\mathrm{DMRG}$ method, and thereby we could not determine the phase boundaries.

What is the most important is that the supersolid states are widely realized between the superfluid and solid states. This is contrast to the results discussed in the single layered model where the supersolid state is not stable at half filling. To clarify how the SS- $1 / 2$ and SS- $\alpha$ states are stabilized at half filling, we show local particle density and superfluid order parameter in Fig. 9] In the SS- $1 / 2$ state $\left(t / V=0.1\right.$ and $\left.t^{\prime} / V=0.3\right)$, the superfluid order parameter is finite at each site. We also find that most of bosons are located at sublattice $\mathrm{A}$ in a certain layer and at sublattices B' and C' in a different one. This feature recalls us the supersolid state in the single layered model away from half filling. In fact, the SS-1/2 state may be regarded as the supersolid state with alternating layered structure, where the supersolid state with $n<1 / 2(>1 / 2)$ is realized in the even (odd) layers. Therefore, we can say that this $\mathrm{SS}-1 / 2$ state in the layered model is stabilized by the self-doping effect through the interlayer hopping. In the other word, the translational symmetry for the particle density in each layer is 
(a) $\mathrm{SS}-1 / 2$

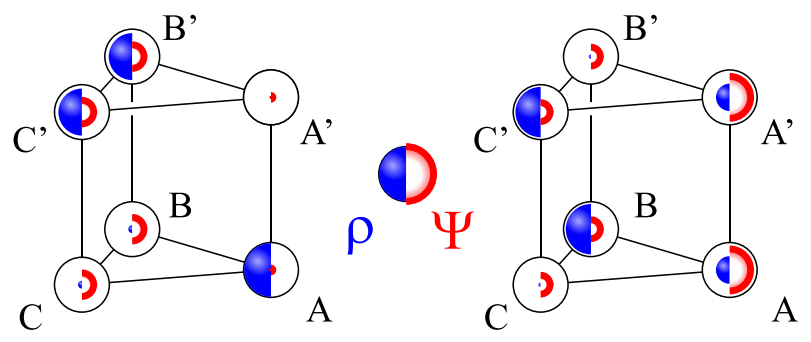

FIG. 9: (Color online) The distributions of the order parameters at the sublattices A, B, C, A', B', and C' in the half-filled system with $t / V=0.1$ when $t^{\prime} / V=0.3$ (a) and 0.5 (b). Radius of left and right semicircles at each sublattice represent a local particle density $\left\langle n_{i m}\right\rangle$ and a local superfluid order parameter $\Psi$, respectively.

spontaneously broken, which stabilizes the $\mathrm{SS}-1 / 2$ state at half filling.

On the other hand, a different mechanism stabilizes the SS- $\alpha$ state. When $t / V=0.1$ and $t^{\prime} / V=0.5$, the $\mathrm{SS}-\alpha$ state is realized with three distinct sublattices in each layer, in contrast to the $\mathrm{SS}-1 / 2$ state, as shown in Fig. 9. At the sublattices A and A', the local particle density is close to half filling and the other sublattices are almost empty or fully-occupied. This enhances superfluid correlations along the chains through the sublattices $A$ and $A^{\prime}$. In fact, the order parameter at each sublattice is maximum and its proximity effect stabilizes the superfluid state in the whole system. On the other hand, the intersite interactions $\left(V, V^{\prime}\right)$ stabilize the alternating structure in the local particle density, where $\mathrm{B}$ and C' (B' and C) sublattices are almost fully-occupied (empty), as shown in Fig. 9 (b). Therefore, we can say that the SS- $\alpha$ state at half filling is mainly stabilized by the one-dimensional network in $z$-direction.
Before closing the section, we would like to comment on experiments on the optical lattice. It is known that the supersolid state in a single layered model has three-fold degeneracy. Therefore, the interlayer coupling should play a crucial role in observing the supersolid state in the layered triangular lattice. In the study, we have clarified that the supersolid states are widely stable at not only the incommensurate fillings but also half filling. Therefore, we can say that ultracold bosons on the layered triangular lattice is one of the most appropriate systems to observe the supersolid states.

\section{SUMMARY}

We have studied ground-state properties in the hardcore Bose-Hubbard model on a layered triangular lattice. Combining cluster mean-field theory with the density matrix renormalization group method, we have discussed how stable the supersolid states realized in a single layer triangular lattice are against the interlayer coupling. We have obtained the rich phase diagram of the system. It has been found that two types of supersolid states are realized even at the commensurate filling.

\section{ACKNOWLEDGMENTS}

The authors would like to thank I. Danshita and D. Yamamoto for valuable discussions. This work was partly supported by Japan Society for the Promotion of Science Grants-in-Aid for Scientific Research Grant Number 25800193 and the Global COE Program "Nanoscience and Quantum Physics" from the Ministry of Education, Culture, Sports, Science and Technology (MEXT) of Japan.
[1] M. H. Anderson, J. R. Ensher, M. R. Matthews, C. E. Wieman, and E. A. Cornell, Science 269, 198 (1995).

[2] I. Bloch and M. Greiner, Adv. At., Mol., Opt. Phys. 52, 1 (2005).

[3] I. Bloch, Nat. Phys. 1, 23 (2005).

[4] D. Jaksch and P. Zoller, Ann. Phys. 315, 52 (2005).

[5] O. Morsch and M. Oberhaler, Rev. Mod. Phys. 78, 179 (2006).

[6] M. Greiner, O. Mandel, T. Esslinger, T. W. Hänsch, and I. Bloch, Nature 415, 39 (2002).

[7] C. Becker, P. Soltan-Panahi, J. Kronjäger, S. Dörscher, K. Bongs, and K. Sengstock, New J. Phys. 12, 065025 (2010).

[8] E. Kim and M. H. W. Chan, Nature 427, 225 (2004); Science 305, 1941 (2004).

[9] H. Matsuda and T. Tsuneto, Prog. Theor. Phys. Suppl. 46, 411 (1970).

[10] W. J. Mullin, Phys. Rev. Lett. 26, 611 (1971).

[11] K.-S. Liu and M. E. Fisher, J. Low Temp. Phys. 10, 655
(1973).

[12] M. Boninsegni, J. Low Temp. Phys. 132, 39 (2003).

[13] S. Wessel and M. Troyer, Phys. Rev. Lett. 95, 127205 (2005).

[14] T. Suzuki and N. Kawashima, Phys. Rev. B 75, 180502 (2007).

[15] S. R. Hassan, L. de Medici, and A.-M. S. Tremblay, Phys. Rev. B 76, 144420 (2007).

[16] X.-F. Zhang, R. Dillenschneider, Y. Yu, and S. Eggert, Phys. Rev. B 84, 174515 (2011).

[17] L. Bonnes and S. Wessel, Phys. Rev. B 84, 054510 (2011).

[18] T. Ohgoe, T. Suzuki, and N. Kawashima, Phys. Rev. Lett. 108, 185302 (2012).

[19] D. Yamamoto, I. Danshita, and C. A. R. Sá de Melo, Phys. Rev. A 85, 021601 (2012).

[20] R. Suzuki and A. Koga, arXiv:1310.0127.

[21] R. T. Scalettar, E. Y. Loh, J. E. Gubernatis, A. Moreo, S. R. White, D. J. Scalapino, R. L. Sugar, and E. Dagotto, Phys. Rev. Lett. 62, 1407 (1989). 
[22] J. K. Freericks, M. Jarrell, and D. J. Scalapino, Phys. Rev. B 48, 6302 (1993).

[23] N. Takemori and A. Koga, J. Phys. Soc. Jpn. 81, 063002 (2012).

[24] A. Koga, T. Higashiyama, K. Inaba, S. Suga, and N. Kawakami, Phys. Rev. A 79, 013607 (2009).

[25] H. P. Büchler and G. Blatter, Phys. Rev. Lett. 91, 130404 (2003).

[26] I. Titvinidze, M. Snoek, and W. Hofstetter, Phys. Rev. Lett. 100, 100401 (2008).

[27] B. D. Metcalf, Phys. Lett. 45A, 1 (1973).

[28] K. Martiyanov, V. Makhalov, and A. Turlapov, Phys. Rev. Lett. 105, 030404 (2010).

[29] T. Oguchi, Prog. Theor. Phys. 13, 148 (1955).

[30] S. R. White, Phys. Rev. Lett. 69, 2863 (1992).
[31] U. Schollwöck, Ann. Phys. 326, 96 (2011).

[32] M. A. Cazalilla, R. Citro, T. Giamarchi, E. Orignac, and M. Rigol, Rev. Mod. Phys. 83, 1405 (2011).

[33] C. N. Yang and C. P. Yang, Phys. Rev. 150, 321 (1966); ibid. 150, 327 (1966); ibid. 151, 258 (1967).

[34] D. Yamamoto, Phys. Rev. B 79, 144427 (2009).

[35] A. Kawaguchi, A. Koga, K. Okunishi, and N. Kawakami, Phys. Rev. B 65, 214405 (2002).

[36] T. Suzuki and S. I. Suga, Phys. Rev. B 70, 054419 (2004).

[37] I. Maruyama, T. Koide, and Y. Hatsugai, Phys. Rev. B 76, 235105 (2007).

[38] D. J. García, K. Hallberg, and M. J. Rozenberg, Phys. Rev. Lett. 93, 246403 (2004). 